\title{
Using Wait-time Thresholds to Improve Mobility: The Case of UberWAV Services in Toronto
}

\author{
Mischa Young ${ }^{1}$ (D), Steven Farber ${ }^{2}$ (D) \\ ${ }^{1}$ Geography and Planning, University of Toronto, ${ }^{2}$ Department of Human Geography, University of Toronto, Scarborough \\ Keywords: ride-hailing, wheelchair accessible vehicles, accessible transportation, wait-time, uberwav \\ https://doi.org/10.32866/001c.14547
}

Transport Findings

\begin{abstract}
We examine the wait-time of Uber's wheelchair accessible service (UberWAV) in Toronto, to determine whether it meets the City's 11-minutes average wait-time requirement. Using a 12-million record dataset of every ride-hailing trip conducted in Toronto between September 2016 and March 2017, we show that wait-times for UberWAV services were, on average, longer during rush hour periods and for trips further away from downtown. Despite this, we find that UberWAV services met the average wait-time requirement imposed by the City and believe that by offering shorter wait-times than previously available, this service significantly improves the mobility of people who require accessible transport services.
\end{abstract}

\section{Research Question and Hypothesis}

Individuals who require accessible transport services typically have fewer mobility options, a condition that can exacerbate wellbeing (Lucas 2004; Páez and Farber 2012; Wasfi, Steinmetz-Wood, and Levinson 2017). Ride-hailing services exhibit potential to improve their mobility; much like taxis, drivers can help passengers that require assistance, and vehicles can be equipped to handle wheelchairs or other mobility devices. However, to date, the reliability and availability of accessible ride-hailing services have achieved varying levels of performance, especially when compared to mainstream services like UberX (Ward 2017; Young and Farber 2020), which has led governments to explore regulatory solutions.

In Toronto, Private Transportation Companies with more than 500 vehicles are required to provide wheelchair accessible services to the public and ensure that they are made available within 11-minutes, the average wait-time for nonaccessible taxis (City of Toronto 2016). This regulation is part of Toronto's broader commitment to "building an inclusive society and providing an accessible environment in which all individuals have access to the City's services and programs in a way that respects the dignity and independence of people with disabilities" (City of Toronto 2009). If met, this laudable goal would represent a considerable mobility improvement for those who require accessible transport services and would contribute to reducing transport inequalities by ensuring similar wait-times between accessible and nonaccessible on-demand services. 
In this paper we test whether Uber's wheelchair accessible service, UberWAV, meets the City of Toronto's average wait-time requirement, and examine how the wait-time for UberWAV services varies across time and space.

\section{Methods and Data}

Data are drawn from a 12-million record dataset of every ride-hailing trip conducted in Toronto between September $7^{\text {th }}, 2016$ and March 31 $1^{\text {st }}, 2017$. The data is provided directly to the city of Toronto by Private Transportation Companies as part of their compliance with the city's Vehicle-for-Hire bylaw (City of Toronto 2020). The dataset includes information on the type of ridehailing service (e.g. UberWAV, UberPOOL, UberX), trip-end locations, and time of trips. Wait-times are defined as the time between the passenger's request and the driver's arrival at the pickup location. It is worth noting that Uber was the only ride-hailing company operating in Toronto during our study period.

UberWAV services are contingent on the availability of a pre-existing supply of wheelchair accessible vehicles. To provide wheelchair accessible services in Toronto, Uber relies on two different groups of drivers that it pairs with passengers through its online platform: 1) drivers operating accessible taxi vehicles; and 2) drivers associated with Dignity Transportation, a specialized accessible transportation provider (Uber 2015). Over the 7-month period of our study, there are a total of 21,493 UberWAV trips (roughly 104 trips per day), of which $15 \%$ are provided by partnering accessible taxis and $85 \%$ are conducted by Dignity Transportation.

\section{Findings}

Figure 1 presents boxplots of the distributions of wait-times for the two UberWAV services, as well as UberX and UberPool for comparison. The yellow dots in the figure represent the mean wait-times, and the red line depicts the 11-minutes threshold under which the average wait-time of UberWAV services must fall. Readers may be accustomed to seeing inferential testing at this point, but it is unnecessary in this context as we are working with population data rather than samples. While longer than UberX and even UberPOOL, which are shown to take 5.2 and 6.4 minutes respectively (Young, Farber, and Palm, forthcoming), the average wait-time for UberWAV services remains at 8 minutes, well below the 11-minutes mark. Overall, 79.3\% of UberWAV trips achieve the commendable wait-time objective imposed by the City of Toronto, and a closer examination uncovers that trips offered by the specialized accessible transportation provider have, on average, shorter wait-times (7.7 minutes) than those offered by the partnering accessible taxi drivers (10.1 minutes). Despite these longer wait-times, partnering accessible taxis play a crucial role in ensuring UberWAV services meet the city-imposed wait time requirement, as they increase the supply of wheelchair accessible vehicles and reduce the burden on other UberWAV providers (Ward 2017). 


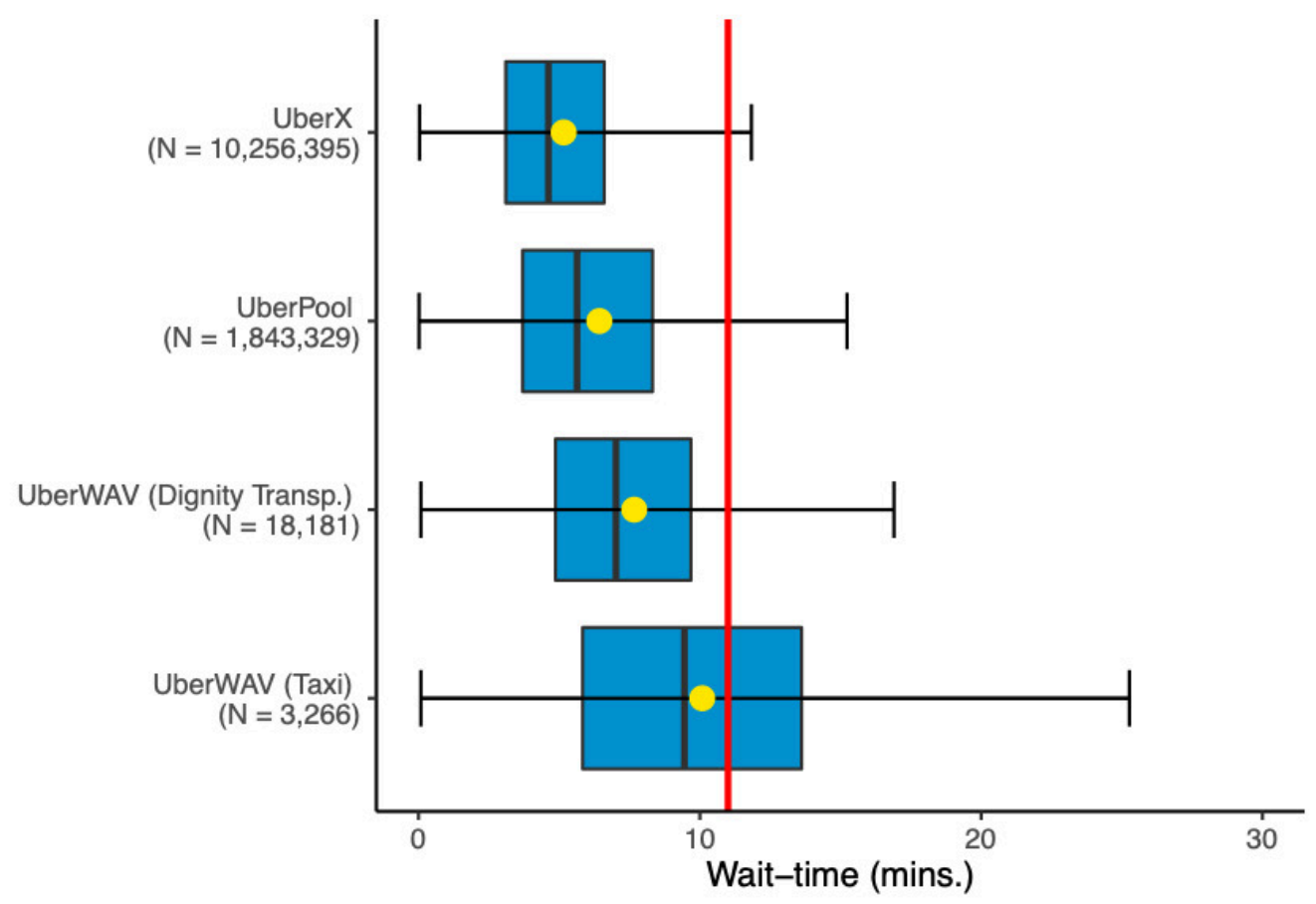

Figure 1 The dispersion of wait-times per ride-hailing service in Toronto ${ }^{1}$

${ }^{1}$ For clarity purposes, outliers are omitted from Figure 1 but are included in the average wait-time calculations.

In Figure 2, we group UberWAV trips into categories based on start-times and examine how wait-times vary throughout the day. Once again, we do not present inferential tests in our analysis as we are working with population data. We find the wait-time of UberWAV trips to be longer, on average, during the Morning and Afternoon periods, which is likely due to higher levels of congestion during those times. The variability in wait-times is also most pronounced during the afternoon period, which we also attribute to higher levels of congestion. Demand is fairly consistent throughout the day, suggesting that it is not the cause for temporal differences in wait-time. Notwithstanding some variability, the city-imposed average wait-time criterion for UberWAV services appears to be met throughout the day.

Figures $3 \mathrm{a}$ and $3 \mathrm{~b}$ display the demand, understood as the number of trips observed in our study period (3a), and spatial variability in wait-times (3b) for UberWAV services in Toronto, aggregated per Traffic Analysis Zones (TAZ). While many TAZs are shown to have wait-times between $9-11$ minutes, very few fail to meet the city-imposed wait-time condition, especially in and around downtown, where demand for UberWAV services is highest. This suggests that demand may be negatively associated with wait-times.

To further establish whether the location of UberWAV trips may have an impact on wait-times, we single-out UberWAV trips that experienced a longer than the city-imposed 11-minute wait-time criterion, and compare their location and start-time characteristics to those of other ride-hailing trips in our sample (see Table 1). We find that UberWAV trips with long wait-times 


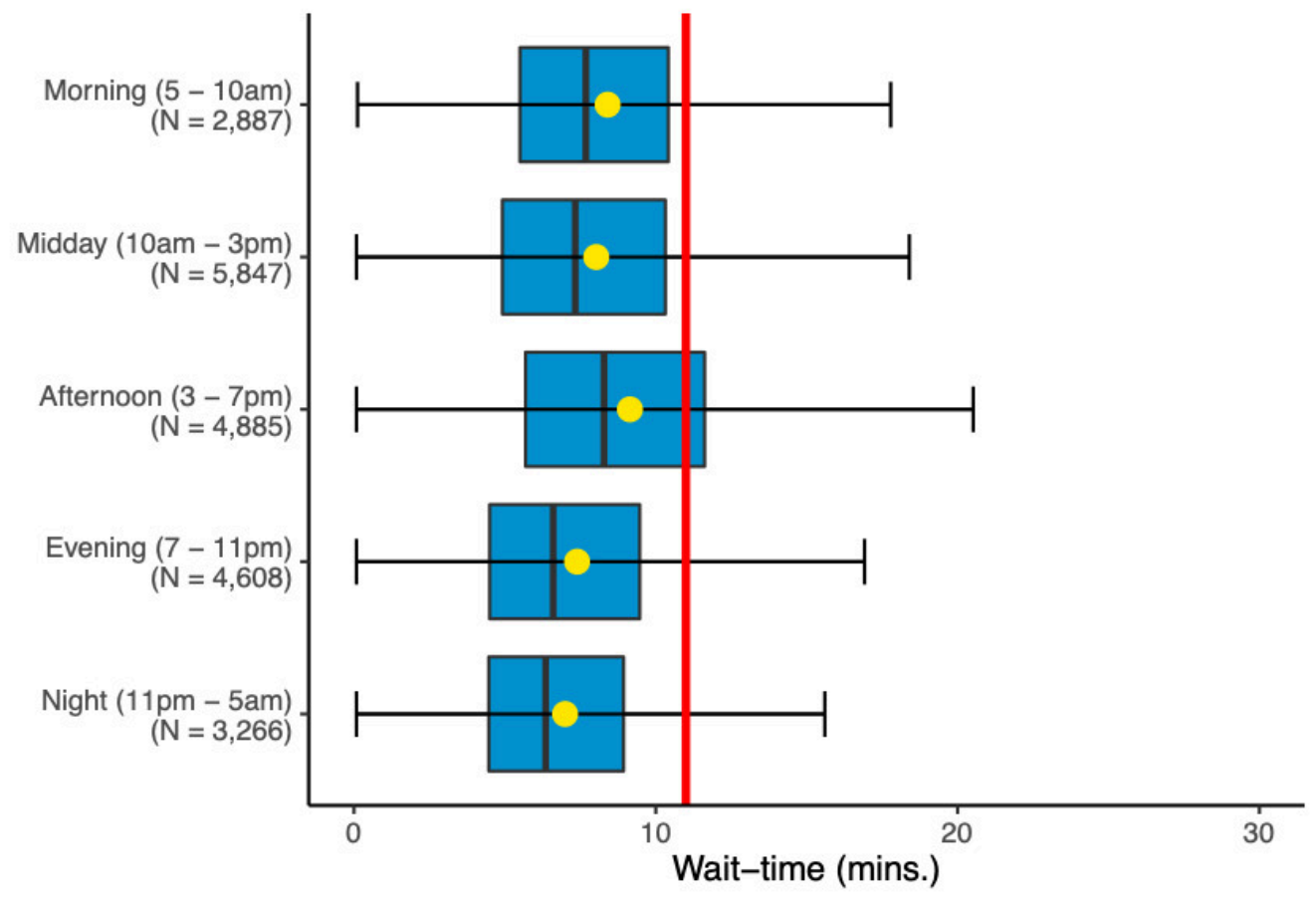

Figure 2 UberWAV wait-times per time of day in Toronto

(> 11-minutes) are more likely to take place in the Afternoon period (31.4\%) and less likely to occur at Night (9.7\%), in comparison to other ride-hailing services including UberWAV trips with short wait-times ( $\geq 11$-minutes). This aligns with the temporal wait-time differences established in Figure 2 and corroborates the likely impact of congestion. Policies should accordingly be designed to provide reliable wait-times to UberWAV users and could include granting high-occupancy vehicle lane access to UberWAV drivers in between pickups to enable them to avoid periodic congestion. The distance to City Hall is further found to be positively correlated with wait-times, as UberWAV trips with wait-time $>11$ minutes are more likely to start further away from downtown, whereas those with wait-times $\geq 11$ minutes tend to occur near downtown. This is likely due to the higher level of UberWAV demand near downtown (see Figures $3 \mathrm{a}$ and $3 \mathrm{~b}$ ) and to the ability of drivers to converge to these areas in order to satisfy demand. The resulting imbalance in supply may lead to longer wait-times in outer areas of the city that do not currently have sufficient UberWAV demand. Faced with this challenge, governments may need to impose a regulation, similar to the existing wait-time criterion, to limit the level of wait-time discrepancy between inner and outer areas of the city. At the very least, they should consider subsidizing UberWAV trips outside of downtown to ensure a sufficient level of supply, as drivers rightfully recognize that they may earn more revenue by commuting downtown and will need to be compensated if we wish to persuade them to remain in outer areas of the city. 


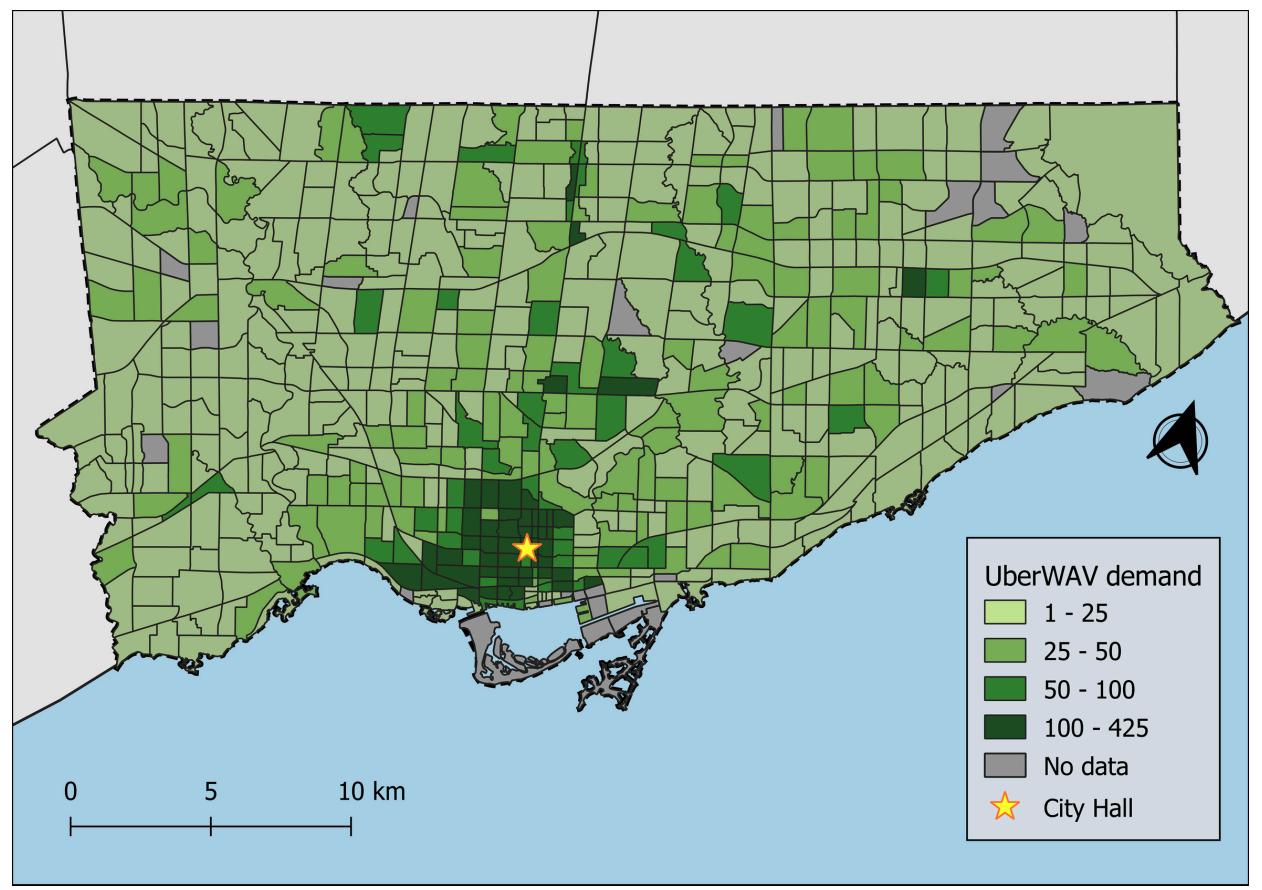

Figure 3a UberWAV demand per traffic analysis zone in Toronto

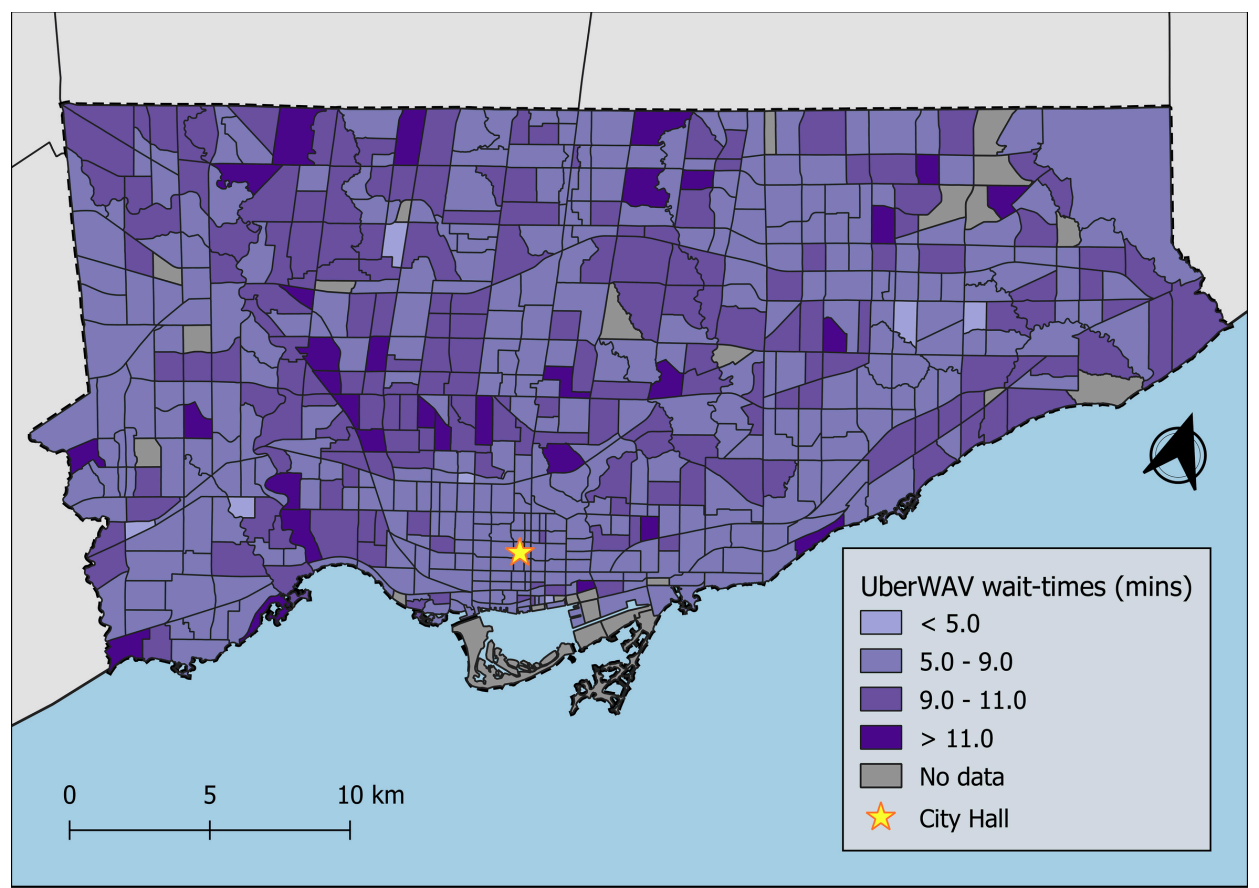

Figure $3 \mathbf{b}$ UberWAV average wait-time per traffic analysis zone in Toronto

Overall, the average wait-time for UberWAV trips in Toronto remains short (8 minutes), especially given that the average wait-time for non-accessible taxicab services in the city is 11 minutes and is presumably even longer for accessible taxis. Our study shows that throughout the day and in most parts of the city, UberWAV services meet the average wait-time requirements imposed by the City of Toronto. Longer wait times are more likely to occur in the afternoon 
Table 1 Characteristics of UberWAV trips that do not meet the city-imposed 11-minutes average wait-time criterion

\begin{tabular}{|c|c|c|c|c|c|}
\hline & & $\begin{array}{r}\text { Long wait } \\
\text { UberWAV } \\
\text { trips }^{\mathrm{a}}\end{array}$ & $\begin{array}{r}\text { Short wait } \\
\text { UberWAV } \\
\text { trips }\end{array}$ & All UberPool & All UberX \\
\hline \multirow{6}{*}{ Start-time } & Morning (5 - 10am) & 14.5 & 13.1 & 14.2 & 15.2 \\
\hline & Midday (10am - 3pm) & 27.4 & 27.2 & 18.4 & 20.6 \\
\hline & Afternoon (3 - 7pm) & 31.4 & 20.5 & 21.5 & 20.1 \\
\hline & Evening (7 - 11pm) & 16.9 & 22.6 & 23.2 & 22.8 \\
\hline & Night (11pm - 5am) & 9.7 & 16.6 & 22.7 & 21.3 \\
\hline & Total & 100.0 & 100.0 & 100.0 & 100.0 \\
\hline \multirow{4}{*}{$\begin{array}{l}\text { Distance to } \\
\text { downtown } \\
\text { City Hall }\end{array}$} & $<2.5 \mathrm{~km}$ & 31.7 & 42.9 & 37.5 & 41.2 \\
\hline & 2.5 to $10 \mathrm{~km}$ & 36.1 & 27.7 & 39.6 & 35.4 \\
\hline & $>10 \mathrm{~km}$ & 32.3 & 29.4 & 23.0 & 23.5 \\
\hline & Total & 100.0 & 100.0 & 100.0 & 100.0 \\
\hline
\end{tabular}

${ }^{a}$ Long wait UberWAV trips are those with wait-times > 11-minutes; Short wait UberWAV trips are those with wait-times $\leq 11$-minutes.

period and away from Downtown. Given the success of this policy, we encourage other cities to introduce similar requirements. Imposing a waittime threshold on accessible ride-hailing trips provides those depending on accessible modes of transportation with a more reliable on-demand service and reduces transport inequalities by ensuring that all users experience similar waittimes.

\section{Acknowledgment}

The authors would like to thank the Fonds de Recherche du Québec - Société et Culture (FRQSC) for funding this research project. 


\section{REFERENCES}

City of Toronto. 2009. Statement of Commitment to Creating an Accessible City. https://www.toronto.ca/city-government/accessibility-human-rights/accessibility-at-the-city-oftoronto/accessible-customer-service/commitment-to-creating-an-accessible-city/.

- - . 2016. A New Vehicle-for-Hire Bylaw to Regulate Toronto's Ground Transportation Industry. https://www.toronto.ca/legdocs/mmis/2016/ls/bgrd/backgroundfile-91911.pdf.

- - . 2020. Vebicle-for-Hire Bylaw Updates. https://www.toronto.ca/city-government/ accountability-operations-customer-service/long-term-vision-plans-and-strategies/vehicle-for-hire/ vehicle-for-hire-bylaw/vehicle-for-hire-bylaw-updates.

Lucas, K. 2004. “Transport and Social Exclusion.” In Running on Empty: Transport, Social Exclusion and Environmental Justice, edited by K. Lucas. Bristol, UK: Policy Press.

Páez, A., and S. Farber. 2012. "Participation and Desire: Leisure Activities among Canadian Adults with Disabilities." Transportation 39 (6): 1055-78. https://doi.org/10.1007/s11116-012-9385-x.

Uber. 2015. "Improving Accessible Transportation Options in Toronto." https://www.uber.com/ en-CA/blog/toronto/accessible-transportation-in-toronto/.

Ward, D. 2017. "Transportation Network Companies \& Accessibility: How Other Jurisdictions Are Navigating Accessibility Issues in an Evolving Vehicle-For Hire Industry \& Ideas for BC.” Doctoral dissertation, University of British Columbia.

Wasfi, R., M. Steinmetz-Wood, and D. Levinson. 2017. "Measuring the Transportation Needs of People with Developmental Disabilities: A Means to Social Inclusion.” Disability and Health Journal 10 (2): 356-60. https://doi.org/10.1016/j.dhjo.2016.10.008.

Young, M., and S. Farber. 2020. "Ride-Hailing Platforms Are Shaping the Future of Mobility, but for Whom?" In The Platform Economy and the City: Urban Peril and Promise in the New Digital Economy, edited by Austin Zwick and Zachary Spicer. McGill-Queens University Press.

Young, M., S. Farber, and M. Palm. Forthcoming. "The True Cost of Sharing: A Detour Penalty Analysis between UberPool and UberX Trips in Toronto." 